\title{
Central Nervous System Kaposi Sarcoma
}

National Cancer Institute

\section{Source}

National Cancer Institute. Central Nervous System Kaposi Sarcoma. NCI Thesaurus.

Code C7006.

A Kaposi sarcoma arising from the brain, spinal cord, or meninges. 